\title{
Generator Columns and High Pressure Liquid Chromatography for Determining Aqueous Solubilities and Octanol-Water Partition Coefficients of Hydrophobic Substances
}

\author{
Howard DeVoe, * Michele M. Miller, $†$ and Stanley P. Wasik $\dagger$ \\ National Bureau of Standards, Washington, DC 20234
}

July 16, 1980

\begin{abstract}
Generator columns packed with a solid support and loaded with a liquid organic phase make it possible to rapidly and conveniently equilibrate water with the organic phase. By coupling the generator column to an extractor column for high pressure liquid chromatographic analysis of the aqueous solution, errors from surface adsorption and loss to the atmosphere are avoided. Using this method, the mean values and confidence limits at a 95 percent confidence level of the aqueous solubility, $S$, and the octanol-water partition coefficient, $P$, of n-propylbenzene at $25^{\circ} \mathrm{C}$ were found to be $S=(4.32 \pm 0.02) \times 10^{-4} M$ and $\log P=3.720 \pm 0.003$.
\end{abstract}

Key words: generator column; hydrophobic; octanol; partition coefficient; propylbenzene; solubility; solute; water.

\section{Introduction}

Measurements of the aqueous solubilities of hydrophobic organic substances are important for understanding hydrophobic interactions in water $[1,2]^{1}$ and for predicting the distribution of pollutants in aquatic environments [3]. In the case of organic liquids, solubilities are often determined by a "shake-flask" method in which one shakes the liquid with water, allows the two phases to separate, and measures the concentration of the organic substance in the aqueous phase by an appropriate analytical technique.

A closely related type of measurement is the partition coefficient of a hydrophobic substance, describing its equilibrium distribution between immiscible organic and aqueous phases. Octanol-water partition coefficients have been used to predict the extent of bioconcentration of organic pollutants in trout muscle [4], and for making other chemical and biological correlations [5]. Partition coefficients are usually determined by the shake-flask method.

In any shake-flask experiment with a hydrophobic substance, the solute concentration in the aqueous phase is low. Consequently the measurement of this concentration can be seriously affected by the presence of colloidal dispersions

-Permanent address: Department of Chemistry, University of Maryland, College Park, Maryland 20742.

tCenter for Chemical Physics, National Measurement Laboratory.

' Figures in brackets indicate literature references at the end of this paper. (emulsions), adsorption of the solute onto surfaces of transfer vessels, and loss of a volatile solute into the atmosphere. These errors can be reduced, but only at a cost of inconvenience and increased time, by centrifuging or replacing the shaking by gentle stirring; prerinsing the glassware with the aqueous phase; and avoiding exposure of the aqueous phase to the atmosphere. A rapid, convenient method for preparing equilibrated aqueous phases without significant errors from such effects would be desirable.

For this purpose we have adapted the generator column method described by May et al [6]. A generator column has a solid support coated with a solid or liquid stationary phase. When water is pumped through the column, an aqueous solution is generated which is equilibrated with the stationary phase. May et al $[6,7]$ used their generator columns, packed with glass beads, mainly to measure solubilities of solid polycyclic aromatic hydrocarbons; the only liquid stationary phase used by them was benzene [7]. We have redesigned the generator column, changed the support to silanized silica in order to increase the coverage, and extended the stationary phase to hydrocarbon-octanol mixtures.

In this paper we describe the use of generator columns containing either propylbenzene (a liquid) for measuring the aqueous solubility, or a dilute solution of propylbenzene in octanol for measuring the octanol-water partition coefficient. For validation of the method, we also obtained data 
using the more conventional stir-flask method. Propylbenzene concentrations were measured by high pressure liquid chromatography (HPLC) on a reverse phase analytical column with ultraviolet detection. The octanol phase was introduced into the chromatograph with a loop injector. For analysis of the aqueous phase, the solute was extracted onto an extractor column and eluted onto the analytical column by the HPLC mobile phase. The combination of a generator column, extractor column, and HPLC analysis is the same as the "dynamic coupled column liquid chromatographic" technique described by May et al [6].

\section{Experimental section}

\subsection{Reagents}

The n-propylbenzene $(99.9 \%)$ and n-nonylbenzene $(99 \%)$ were obtained from Chemical Samples Co., ${ }^{2}$ Columbus, Ohio. The n-octanol (Fisher Scientific Co., Certified grade) was washed successively with $0.1 \mathrm{M} \mathrm{H}_{2} \mathrm{SO}_{4}, 0.1 \mathrm{M} \mathrm{NaOH}$, and distilled water, it was then dried with $\mathrm{CaCl}_{2}$, filtered and distilled at atmospheric pressure. HPLC grade methanol and water (Baker) were used to prepare the mobile phases for liquid chromatography. Ordinary distilled water was used for other aqueous solutions.

\subsection{Generator Columns}

The design of a typical generator column is shown in figure 1. The column consists of a $6 \mathrm{~mm}$ (outside diameter) Pyrex tube joined to a short enlarged section of $9 \mathrm{~mm}$ Pyrex tubing. To pack the column a plug of silanized glass wool was inserted at one end of the $6 \mathrm{~mm}$ tube. A silanized diatomaceous silica support (about $0.5 \mathrm{~g}$ of 100-120 mesh Chromosorb W, high performance grade, cleaned by Soxhlet extraction with ethanol and dried) was poured into the tube with tapping and retained with a second plug of silanized glass wool.

The column was loaded by pulling the liquid organic phase, propylbenzene or a propylbenzene-octanol mixture, through the dry support with gentle suction. Each propylbenzene-octanol mixture had previously been equilibrated with water using gentle stirring for several hours, and a portion of the equilibrated mixture was analyzed by HPLC.

After it was loaded, the column was set up vertically with its inlet end at the bottom and a Teflon outlet tube attached to the top. Water was pumped into the inlet from a Milton

\footnotetext{
${ }^{2}$ Certain trade names and company products are identified in order to adequately specify the experimental procedure. In no case does such identification imply recommendation or endorsement by the National Bureau of Standards, nor does it imply that the products are necessarily the best available for the purpose.
}

Roy Minipump. The water flow pushed a plug of the organ. ic phase off the support and out the outlet tube. A new outlet tube consisting of clean $1 / 16$ in $(1.6 \mathrm{~mm})$ stainless steel tubing was then attached to the column with a clean $1 / 4$ in 6 $\mathrm{mm}$ ) stainless steel column end fitting containing a $5-\mu \mathrm{m}$ frit to protect the switching valve of the HPLC system (see sec. 2.4). During the generation of the aqueous solution, the column was inverted from its previous position to put the inlet at the top with the water flowing downward through the support, as shown in figure 1 . The purpose of the section of larger diameter tubing below the support was to trap any of the lighter density organic phase that was pushed off the support by the water flow, in order to prevent droplets of this phase from being swept into the outlet tube.

Columns were reused by pumping methanol through to remove the organic phase, drying the support with a warm stream of nitrogen, and loading with a new organic phase.
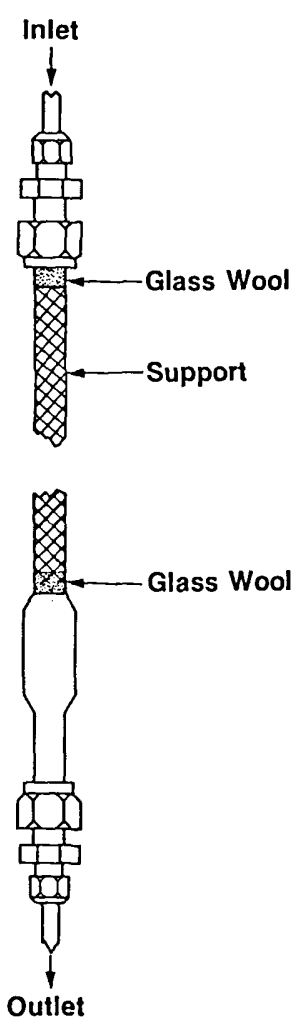

Figure 1. Generator column. The column is constructed from $6 \mathrm{~mm}$ and $9 \mathrm{~mm}$ Pyrex tubing. The length of the support is typically $12 \mathrm{~cm}$. Connections to inlet and outlet tubing are by means of $1 / 4$ in $(6 \mathrm{~mm})$ stainless steel fittings with Teflon ferrules. 


\subsection{Stir-flask equilibrations}

For each equilibration, about $100 \mathrm{~mL}$ of water and about $4 \mathrm{~mL}$ of the organic phase were placed in a closed Pyrex vessel and stirred with a small glass-encased magnetic stirring bar. To prevent emulsion formation in the aqueous phase, vigorous stirring was avoided. Most of the organic layer was confined to a narrow neck at the top of the vessel. Above the neck was $a 3 / 8$ in $(9.5 \mathrm{~mm})$ tube septum fitting for sampling of the organic layer after equilibration. The equilibrated aqueous phase was transferred to the HPLC system, with the aid of nitrogen pressure, through $1 / 16$ in $(1.6 \mathrm{~mm})$ stainless steel tubing attached to a $1 / 4$ in $(6 \mathrm{~mm})$ outlet tube.

\subsection{HPLC System}

The HPLC analytical system is shown schematically in figure 2. Two reciprocating piston pumps delivered the mobile phase (water or a methanol-water mixture) through two 6-port high pressure rotary valves and a $30 \times 0.6 \mathrm{~cm}$ C-18 analytical column to an ultraviolet absorption detector operating at $254 \mathrm{~nm}$. Chromatogram peaks were recorded and integrated with a recording integrator.

One of the 6-port valves was a sample injection valve used for injecting samples of either the octanol phase for analysis, or standard solutions of the solute in methanol for determining response factors. The sample loop volume was determined by a spectrophotometric method using basic chromate solutions which are known to be suitable as absorption standards [8]. The method consisted of measuring the absorbance at $373 \mathrm{~nm}$ of three loopfuls of an aqueous stock solution of $\mathrm{K}_{2} \mathrm{CrO}_{4}$ (1.3\% by weight) diluted to $50 \mathrm{~mL}$ with 0.2 percent $\mathrm{KOH}$, and measuring the absorbance of the same stock solution after diluting 1:500 with 0.2 percent $\mathrm{KOH}$. From these absorbances the loop volume was calculated to be $(35.7 \pm 0.1) \mu \mathrm{L}$.
The other 6-port valve in the system served as a switching valve for the extractor column which was used to remove solute from the aqueous solutions. The extractor column was a $6.6 \times 0.6 \mathrm{~cm}$ stainless steel tube with stainless steel end fittings containing $5-\mu \mathrm{m}$ frits, filled with a superficially porous reverse phase packing (Bondapak $\mathrm{C}_{18}$ /Corasil, Waters Associates). Aqueous solution from a stir-flask vessel or a generator column entered the switching valve through the $1 / 16$ in $(1.6 \mathrm{~mm})$ stainless steel tubing labelled " $A$ " in figure 2 . A water bath was used to thermostat to $\pm 0.05^{\circ} \mathrm{C}$ the generator column (when used), the switching valve, the extractor column, and the interconnecting stainless steel tubing.

The procedure for analyzing the aqueous phase was first to flow the aqueous solution to waste with the switching valve in the inject position in order to equilibrate internal surfaces with the solution, thus insuring that the analyzed sample would not be depleted by solute adsorption on surfaces upstream from the valve. At the same time, water was pumped from the HPLC pumps in order to displace methanol from the extractor column. The switching valve was next changed to the load position to divert a sample of the solution through the extractor column, and the liquid leaving this column was collected in a weighing bottle. During this extraction step, the mobile phase was changed to a methanol-water mixture (generally $65 \%$ methanol pumped at $3 \mathrm{~mL} / \mathrm{min}$ for propylbenzene analysis) to condition the analytical column. After the desired volume of sample had been extracted, the switching valve was returned to the inject position for elution and analysis. Provided that there was no breakthrough of solute from the extractor column during the extraction step, the chromatographic peak represented all of the solute in the sample. The solute concentration in the aqueous phase was calculated from the peak area and the weight of the extracted liquid collected in the weighing bottle.

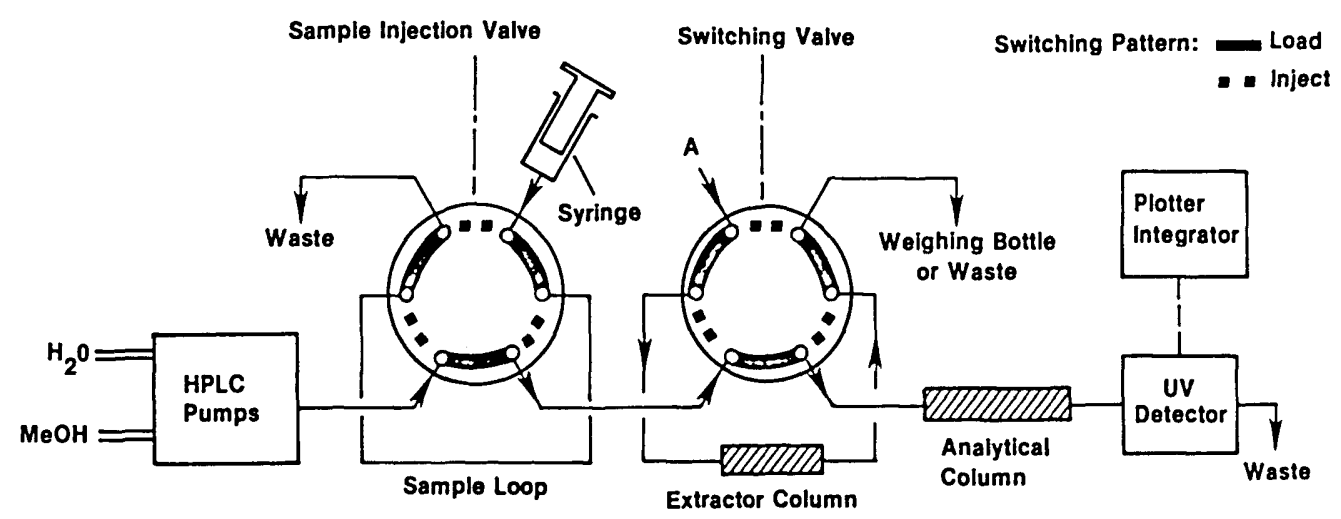

FIGURE 2. HPLC system. Connections shown by thin solid lines are $1 / t_{6}$ in $(1.6 \mathrm{~mm})$ stainless steel tubing. Connection $A$ is attached to the outlet of either a stir-flask vessel or a generator column. 


\section{Results and Discussion}

\subsection{Solubility}

Table I lists our solubility results for propylbenzene. The aqueous samples were $2-6 \mathrm{~mL}$ in volume. The stir-flask and generator column results for comparable temperatures agree within experimental uncertainty. Our value at $25^{\circ} \mathrm{C}$, $4.3 \times 10^{-4} \mathrm{M}$, is similar to the values for this temperature reported by Andrews and Keefer [9] $\left(4.6 \times 10^{-4} \mathrm{M}\right)$ and by Hermann [1] $\left(5.0 \times 10^{-4} \mathrm{M}\right)$. The temperature dependence of the solubility suggests that there is a minimum in the neighborhood of $20^{\circ} \mathrm{C}$. Solubility minima near $18{ }^{\circ} \mathrm{C}$ have been observed for benzene and its methyl, dimethyl, and ethyl derivatives [10]. In the case of propylbenzene, Gill et al [11] predict from the temperature dependence of the heat of solution that there is a solubility minimum at $(18.6 \pm 0.2)^{\circ} \mathrm{C}$ which is consistent with our results.

TABLE I. Aqueous solubility of n-propylbenzene.

\begin{tabular}{ccc}
\hline$\ell^{\circ} \mathrm{C}^{\mathrm{a}}$ & $\mathrm{n}^{\mathrm{b}}$ & $\mathrm{c}_{w} / 10^{-4} \mathrm{M}^{\mathrm{c}}$ \\
\hline $\begin{array}{l}\text { stir-flask, stirred } 16 \mathrm{~h} \\
\text { ambient }\end{array}$ & 4 & $4.27 \pm 0.06$ \\
generator column & & \\
15.0 & 3 & $4.26 \pm 0.05$ \\
20.0 & 2 & $4.25 \pm 0.12$ \\
25.0 & 3 & $4.32 \pm 0.02$ \\
30.0 & 3 & $4.45 \pm 0.05$ \\
\hline
\end{tabular}

- Equilibration temperature. Ambient is $(23 \pm 1)^{\circ} \mathrm{C}$.

- Number of aqueous solution samples analyzed.

-Aqueous concentration; the mean value and the confidence limits at a 95 percent confidence level are listed.

\subsection{Partition coefficient}

The octanol-water partition coefficient, $P$, was calculated from the measured propylbenzene concentrations in the equilibrated octanol and aqueous phases by dividing the concentration in the octanol phase by the concentration in the aqueous phase.

The volumes of the aqueous phase samples used to obtain the partition coefficient values listed in table II were in the range $1-4 \mathrm{~mL}$ for the stir-flask results and $10-24 \mathrm{~mL}$ for the generator column results. The water flow rate through the generator columns was $2 \mathrm{~mL} / \mathrm{min}$. Further experiments with a generator column at $25^{\circ} \mathrm{C}$ showed that within experimental uncertainty (standard deviation $3 \%$ or less) the measured aqueous concentration was independent of the water flow rate (in the range $0.5-2 \mathrm{~mL} / \mathrm{min}$ ) and was independent of the volume of sample passed through the ex-
TABLE II. Octanol-water partition coefficient of n-propylbenzene.

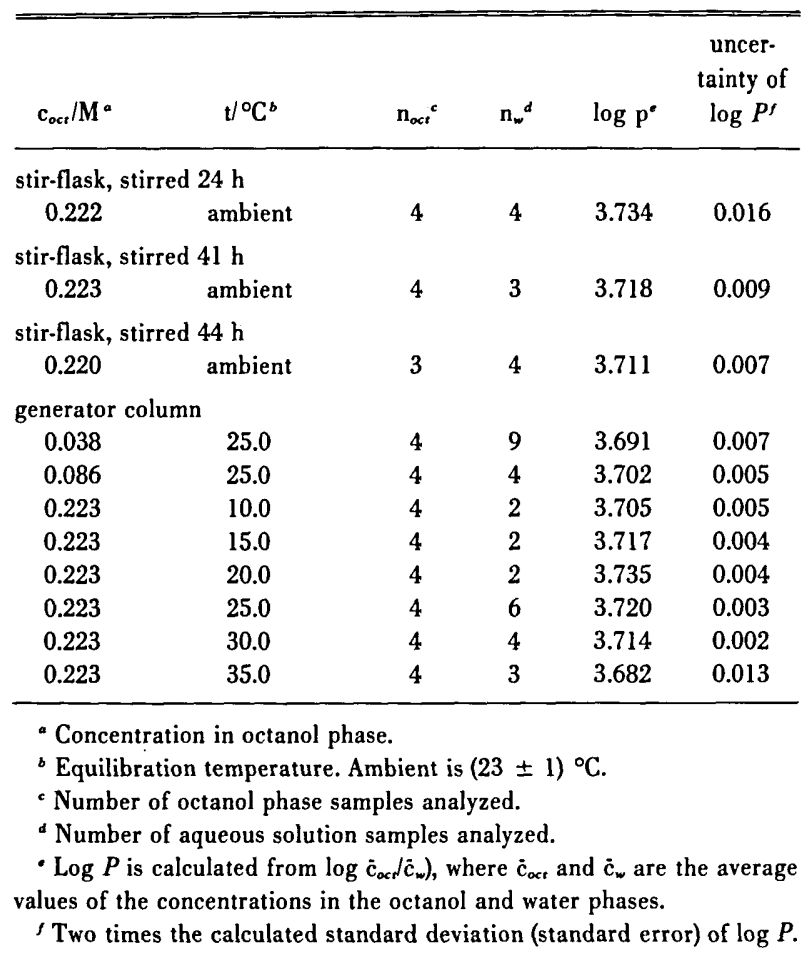

tractor column (in the range $2-24 \mathrm{~mL}$ ). These experiments show that under our experimental conditions the equilibration was complete in the generator column, and that there was no significant breakthrough of propylbenzene from the extractor column.

Three stir-flask equilibrations with different stirring times were carried out (table II). With increased stirring time, there was a decrease in the partition coefficient values of about the same size as the experimental uncertainty. There is good agreement between these values and the generator column results.

The partition coefficient is seen to vary with temperature (table II), having a maximum in the vicinity of $20^{\circ} \mathrm{C}$. It is interesting to note that while the solubility and the partition coefficient of propylbenzene both vary with temperature, their product over the temperature range in which both quantities were measured $\left(15-30^{\circ} \mathrm{C}\right)$ is essentially constant $(2.26 \pm 0.05 \mathrm{M})$. This suggests that the heat of solution of propylbenzene in octanol is small.

The partition coefficient measurements at $25^{\circ} \mathrm{C}$ (table II) show a small but systematic decrease of $\log P$ with decreasing concentration in the octanol phase. Extrapolation to infinite dilution gives $\log P=3.688 \pm 0.006$ at $25^{\circ} \mathrm{C}$. Iwasa et al [12] report $\log P=3.68 \pm 0.01$ from shake-flask measurements at ambient temperature, in excellent agreement with our results. 


\subsection{Colloidal dispersions from generator-columns}

A potentially serious source of systematic error in any determination of the partition coefficient of a hydrophobic solute is the possible formation of colloidal droplets of the organic phase in the aqueous phase due to shear forces during the equilibration. Solute contained in these droplets would tend to make the apparent solute concentration in the aqueous phase higher than the concentration of the solute present in true aqueous solution. The calculated apparent partition coefficient would then be too low.

One may test for this source of error in a generator column by measuring the apparent partition coefficient of a solute which has a much higher partition coefficient than the solute of interest. We carried out such a test with a generator column loaded with a $0.08 \mathrm{M}$ solution of nonylbenzene in octanol. A 25 -mL sample of the aqueous phase from this column gave a chromatogram peak, barely visible above baseline noise, which represented an aqueous phase concentration of at most $1 \times 10^{-7}$ times the octanol phase concentration. The largest volume fraction of the bulk octanol phase which could have been present as droplets in the aqueous phase, then, was $1 \times 10^{-7}$. If a $1 \times 10^{-7}$ volume fraction of octanol phase had also been present in the propylbenzene partition coefficient measurements, the error in the apparent partition coefficient of propylbenzene would have been only 0.05 percent.

The value one would expect for the true partition coefficient of nonylbenzene, based on a linear extrapolation of $\log P$ versus carbon number from data [13] for the homologous series ethyl, n-propyl-, and n-butylbenzene is approximately $1 \times 10^{7}$. Thus the volume fraction of octanol in the aqueous phase must have been even less than $1 \times 10^{-7}$. We concluded that any error from colloidal dispersions in our determination of the partition coefficient of propylbenzene by the generator column method is much less than the uncertainty from random experimental errors.

\subsection{Stripping of the stationary phase from generator columns}

Because the organic stationary phase of a generator column is soluble to some extent in the water passing through the column, there is a continuous removal of the stationary phase. It can be shown from chromatographic theory that the composition of the generated aqueous solution should remain constant until the arrival at the downstream end of the support of a zone in which the stationary phase, or a component of this phase, has been completely stripped. At this point the generator column can no longer be used.

The volume of water required to strip the generator column used to measure the solubility of propylbenzene was much greater (on the order of $10 \mathrm{~L}$ ) than the total volume ot water passed through the column. In the case of a generator column used to determine a partition coefficient, it is the component of the organic phase having the smallest partition coefficient between the organic phase and the water phase which is stripped first. For propylbenzene-octanol mixtures, this component is octanol for which [14] $\log P=$ 3.15. We observed constancy in the propylbenzene concentrations of the aqueous solutions generated by columns loaded with these mixtures, as expected, until about one liter of solution had been generated; then a sudden increase in the concentration occurred. We attribute this increase to the stripping of the last amount of octanol and the saturation of the water with the liquid propylbenzene which remained on the support. The change was obvious and the measurements were discontinued as soon as it occurred.

\subsection{Advantages of generator columns}

Generator columns have several advantages over shakeflask and stir-flask methods for equilibrating water with a liquid organic phase. The flow rate of water through the column can be made slow enough to avoid colloidal dispersions, while the large interfacial area between the organic and aqueous phases allows rapid equilibration. When the column is part of a continuous and closed flow system, the system walls become equilibrated with the aqueous solution and errors from adsorption are avoided; also there is no exposure of the solution to the atmosphere. Only about $1 \mathrm{~mL}$ of the organic phase is sufficient to load the column, and the column will generate samples of the aqueous solution of whatever volume is needed for analysis until the organic phase has been stripped. Once a column has been loaded with a given organic phase, it is a simple matter to vary other parameters such as the temperature or the concentration of an added salt in the aqueous phase.

\section{References}

[1] Hermann, R. B. Theory of hydrophobic bonding. II. The correlation of hydrocarbon solubility in water with solvent cavity surface area. J. Phys. Chem. 76(19): 2754-2759; 1972 September.

[2] Tanford, C. The hydrophobic effect: formation of micelles and biological membranes. New York: John Wiley and Sons; 1973. 710p.

[3] Bobra, A.; Mackay, D.; Shiu, W. Y. Distribution of hydrocarbons among oil, water, and vapor phases during oil dispersant toxicity tests. Bull. Environm. Contam. Toxicol. 23(5): 558-565; 1979 November.

[4] Neely, W. B.; Branson, D. R.; Blau, G. E. Partition coefficient to measure bioconcentration potential of organic chemicals in fish. Environ. Sci. Technol. 8(13): 1113-1115; 1974 December.

[5] Leo, A.; Hansch, C.; Elkins, D. Partition coefficients and their uses. Chem. Rev. 71(6): 525-616; 1971 December. 
[6] May, W. E.; Wasik, S. P.; Freemann, D. H. Determination of the aqueous solubility of polynuclear aromatic hydrocarbons by a coupled column liquid chromatographic technique. Anal. Chem. 50(1): 175-179; 1978 January.

[7] May, W. E.; Wasik, S. P.; Freemann, D. H. Determination of the solubility behavior of some polycyclic aromatic hydrocarbons in water. Anal. Chem. 50(7): 997-1000; 1978 June.

[8] Edisbury, J. R. Practical hints on absorption spectrometry (ultraviolet and visible). New York: Plenum Press; 1967. 266 p.

[9] Andrews, L. J.; Keefer, R. M. Cation complexes of compounds containing carbon-carbon double bonds. VIII. Further studies on the argentation of substituted benzenes. J. Am. Chem. Soc. 72(11): 5034-5037; 1950 November.
[10] Bohon, R. L.; Claussen, W. F. The solubility of aromatic hydrocarbons in water. J. Am. Chem. Soc. 73(4): 1571-1578; 1951 April.

[11] Gill, S. J.; Nichols, N. F.; Wadso, I. Calorimetric determination of enthalpies of solution of slightly soluble liquids. J. Chem. Thermodyn. 8(5): 445-452; 1976 May.

[12] Iwasa, J.; Fujita, T.; Hansch, C. Substituent constants for aliphatic functions obtained from partition coefficients. J. Med. Chem. 8(2): 150-153; 1965 March.

[13] Hansch, C.; Leo, A. Substituent constants for correlation analysis in chemistry and biology. New York: John Wiley and Sons; 1979. $339 p$.

[14] Collander, R. The partition of organic compounds between higher alcohols and water. Acta. Chem. Scand. 5(5): 774-780; 1951. 